\title{
On a new Hardy-Mulholland-type inequality and its more accurate form
}

Aihua $\mathrm{Li}^{\mathrm{i}^{*}}$, Bicheng Yang ${ }^{2}$ and Leping $\mathrm{He}^{1}$

\section{"Correspondence:}

jsdxstxylah@163.com

${ }^{1}$ College of Mathematics and

Statistics, Jishou University, Jishou,

Hunan 416000, P.R. China

Full list of author information is

available at the end of the article

\begin{abstract}
Using weight coefficients and applying the well-known Hermite-Hadamard inequality, a new Hardy-Mulholand-type inequality with a best possible constant factor is given. Furthermore, we also consider the more accurate equivalent forms, the operator expressions and some particular inequalities. The lemmas and theorems provide an extensive account of this type of inequalities.
\end{abstract}

MSC: 26D15; 47A07

Keywords: Hardy-Mulholand-type inequality; weight coefficient; equivalent form; reverse; operator

\section{Introduction}

Assuming that $p>1, \frac{1}{p}+\frac{1}{q}=1, a_{m}, b_{n} \geq 0, a=\left\{a_{m}\right\}_{m=1}^{\infty} \in l^{p}, b=\left\{b_{n}\right\}_{n=1}^{\infty} \in l^{q},\|a\|_{p}=$ $\left(\sum_{m=1}^{\infty} a_{m}^{p}\right)^{\frac{1}{p}}>0,\|b\|_{q}>0$, we have the following Hardy-Hilbert inequality with the best possible constant factor $\frac{\pi}{\sin (\pi / p)}(c f .[1]$, Theorem 315):

$$
\sum_{m=1}^{\infty} \sum_{n=1}^{\infty} \frac{a_{m} b_{n}}{m+n}<\frac{\pi}{\sin (\pi / p)}\|a\|_{p}\|b\|_{q}
$$

The more accurate inequality of (1) is given as follows (cf. [2] and Theorem 323 of [1]):

$$
\sum_{m=1}^{\infty} \sum_{n=1}^{\infty} \frac{a_{m} b_{n}}{m+n-\alpha}<\frac{\pi}{\sin (\pi / p)}\|a\|_{p}\|b\|_{q} \quad(0 \leq \alpha \leq 1)
$$

which is an extension of (1). We still have the following Mulholland inequality similar to (1) with the same best possible constant factor $\frac{\pi}{\sin (\pi / p)}(c f$. [3] or Theorem 343 of [1], replacing $\frac{a_{m}}{n}, \frac{b_{n}}{n}$ by $\left.a_{m}, b_{n}\right)$ :

$$
\sum_{m=2}^{\infty} \sum_{n=2}^{\infty} \frac{a_{m} b_{n}}{\ln m n}<\frac{\pi}{\sin (\pi / p)}\left(\sum_{m=2}^{\infty} \frac{a_{m}^{p}}{m^{1-p}}\right)^{\frac{1}{p}}\left(\sum_{n=2}^{\infty} \frac{b_{n}^{q}}{n^{1-q}}\right)^{\frac{1}{q}} .
$$

Inequalities (1)-(3) are important in analysis and applications (cf. [2, 4-9]).

(c) $2016 \mathrm{Li}$ et al. This article is distributed under the terms of the Creative Commons Attribution 4.0 International License (http://creativecommons.org/licenses/by/4.0/), which permits unrestricted use, distribution, and reproduction in any medium, provided you give appropriate credit to the original author(s) and the source, provide a link to the Creative Commons license, and indicate if changes were made. 


$$
\text { If } \begin{aligned}
\mu_{i}, v_{j} & >0(i, j \in \mathbf{N}=\{1,2, \ldots\}), \\
U_{m} & :=\sum_{i=1}^{m} \mu_{i}, \quad V_{n}:=\sum_{j=1}^{n} v_{j} \quad(m, n \in \mathbf{N}),
\end{aligned}
$$

then we have the following Hardy-Hilbert-type inequality ( $c f$. Theorem 321 of [1], replacing $\mu_{m}^{1 / q} a_{m}$ and $v_{n}^{1 / p} b_{n}$ by $a_{m}$ and $b_{n}$ ):

$$
\sum_{m=1}^{\infty} \sum_{n=1}^{\infty} \frac{a_{m} b_{n}}{U_{m}+V_{n}}<\frac{\pi}{\sin \left(\frac{\pi}{p}\right)}\left(\sum_{m=1}^{\infty} \frac{a_{m}^{p}}{\mu_{m}^{p-1}}\right)^{\frac{1}{p}}\left(\sum_{n=1}^{\infty} \frac{b_{n}^{q}}{v_{n}^{q-1}}\right)^{\frac{1}{q}} .
$$

For $\mu_{i}=v_{j}=1(i, j \in \mathbf{N})$, (5) reduces to (1).

In 2015, Yang [10] gave an extension of (5) as follows: For $0<\lambda_{1}, \lambda_{2} \leq 1, \lambda_{1}+\lambda_{2}=\lambda$, $\left\{\mu_{m}\right\}_{m=1}^{\infty}$, and $\left\{v_{n}\right\}_{n=1}^{\infty}$ are decreasing, and $U_{\infty}=V_{\infty}=\infty$, we have the following inequality with the best possible constant factor $B\left(\lambda_{1}, \lambda_{2}\right)$ :

$$
\sum_{m=1}^{\infty} \sum_{n=1}^{\infty} \frac{a_{m} b_{n}}{\left(U_{m}+V_{n}\right)^{\lambda}}<B\left(\lambda_{1}, \lambda_{2}\right)\left[\sum_{m=1}^{\infty} \frac{U_{m}^{p\left(1-\lambda_{1}\right)-1} a_{m}^{p}}{\mu_{m}^{p-1}}\right]^{\frac{1}{p}}\left[\sum_{n=1}^{\infty} \frac{V_{n}^{q\left(1-\lambda_{2}-1\right)} b_{n}^{q}}{v_{n}^{q-1}}\right]^{\frac{1}{q}},
$$

where $B(u, v)$ is the beta function defined by $(c f .[11])$

$$
B(u, v):=\int_{0}^{\infty} \frac{t^{u-1}}{(1+t)^{u+v}} d t \quad(u, v>0)
$$

In a similar way, Huang and Yang [12] gave a more accurate inequality of (6) and Yang and Chen [13] obtained a Hardy-Hilbert-type inequality with another kernel and a best possible constant factor.

In this paper, using the way of weight coefficients and applying Hermite-Hadamard's inequality, a Hardy-Mulholland-type inequality with a best possible constant factor similar to (6) is proved, which is an extension of (3). Furthermore, the more accurate HardyMulholland-type inequality is built by introducing a few parameters. We also consider the equivalent forms, the operator expressions and some particular inequalities.

\section{Some lemmas and an example}

In the following of this paper, we assume that $p>1, \frac{1}{p}+\frac{1}{q}=1, \mu_{i}, v_{j}>0(i, j \in \mathbf{N})$, with $\mu_{1}=v_{1}=1, U_{m}$ and $V_{n}$ are indicated by (4), $\alpha \leq \frac{\mu_{2}}{2}, \beta \leq \frac{v_{2}}{2}, 0<\lambda_{1}, \lambda_{2} \leq 1, \lambda_{1}+\lambda_{2}=\lambda$, $a_{m}, b_{n} \geq 0,\|a\|_{p, \Phi_{\lambda}}:=\left(\sum_{m=2}^{\infty} \Phi_{\lambda}(m) a_{m}^{p}\right)^{\frac{1}{p}}$, and $\|b\|_{q, \Psi_{\lambda}}:=\left(\sum_{n=2}^{\infty} \Psi_{\lambda}(n) b_{n}^{q}\right)^{\frac{1}{q}}$, where

$$
\begin{aligned}
& \Phi_{\lambda}(m):=\frac{\left[\ln \left(U_{m}-\alpha\right)\right]^{p\left(1-\lambda_{1}\right)-1}}{\left(U_{m}-\alpha\right)^{1-p} \mu_{m+1}^{p-1}}, \\
& \Psi_{\lambda}(n):=\frac{\left[\ln \left(V_{n}-\beta\right)\right]^{q\left(1-\lambda_{2}\right)-1}}{\left(V_{n}-\beta\right)^{1-q} v_{n+1}^{q-1}} \quad(m, n \in \mathbf{N} \backslash\{1\}) .
\end{aligned}
$$

Lemma 1 Suppose that $a \in \mathbf{R}, f(x)$ in continuous in $\left[a-\frac{1}{2}, a+\frac{1}{2}\right], f^{\prime}(x)$ is strictly increasing in $\left(a-\frac{1}{2}, a\right)$ and $\left(a, a+\frac{1}{2}\right)$, and $f^{\prime}(a-0) \leq f^{\prime}(a+0)$. We have the following Hermite- 
Hadamard inequality (cf. Lemma 1 of [14]):

$$
f(a)<\int_{a-\frac{1}{2}}^{a+\frac{1}{2}} f(x) d x
$$

Example 1 Assuming that $\left\{\mu_{m}\right\}_{m=1}^{\infty}$ and $\left\{v_{n}\right\}_{n=1}^{\infty}$ are decreasing, we set $\mu(t):=\mu_{m}, t \in(m-$ $1, m](m \in \mathbf{N}) ; v(t):=v_{n}, t \in(n-1, n](n \in \mathbf{N})$,

$$
U(x):=\int_{0}^{x} \mu(t) d t \quad(x \geq 0), \quad V(y):=\int_{0}^{y} v(t) d t \quad(y \geq 0) .
$$

Then we have $U(m)=U_{m}, V(n)=V_{n}, U(\infty)=U_{\infty}, V(\infty)=V_{\infty}$ and

$$
\begin{aligned}
& U^{\prime}(x)=\mu(x)=\mu_{m}, \quad x \in(m-1, m), \\
& V^{\prime}(y)=v(y)=v_{n}, \quad y \in(n-1, n)(m, n \in \mathbf{N}) .
\end{aligned}
$$

For fixed $m, n \in \mathbf{N} \backslash\{1\}$, we define the function $h(x)$ as follows:

$$
h(x):=\frac{\ln ^{\lambda_{2}-1}(V(x)-\beta)}{(V(x)-\beta)\left[\ln \left(U_{m}-\alpha\right)+\ln (V(x)-\beta)\right]^{\lambda}}, \quad x \in\left[n-\frac{1}{2}, n+\frac{1}{2}\right] .
$$

Then $h(x)$ in continuous in $\left[n-\frac{1}{2}, n+\frac{1}{2}\right]$, and, for $x \in\left(n-\frac{1}{2}, n\right)(n \in \mathbf{N} \backslash\{1\})$,

$$
\begin{aligned}
h^{\prime}(x)= & -\left\{\frac{\ln ^{\lambda_{2}-1}(V(x)-\beta)}{(V(x)-\beta)}+\frac{\lambda \ln ^{\lambda_{2}-1}(V(x)-\beta)}{\ln \left[\left(U_{m}-\alpha\right)(V(x)-\beta)\right]}\right. \\
& \left.+\frac{1-\lambda_{2}}{(V(x)-\beta)^{2-\lambda_{2}}}\right\} \frac{v_{n}}{(V(x)-\beta) \ln ^{\lambda}\left[\left(U_{m}-\alpha\right)(V(x)-\beta)\right]} .
\end{aligned}
$$

In view of $1-\lambda_{2} \geq 0, h^{\prime}(x)(<0)$ is strictly increasing in $\left(n-\frac{1}{2}, n\right)$ and

$$
\begin{aligned}
\lim _{x \rightarrow n-} h^{\prime}(x)= & h^{\prime}(n-0)=-\left\{\frac{\ln ^{\lambda_{2}-1}\left(V_{n}-\beta\right)}{\left(V_{n}-\beta\right)}+\frac{\lambda \ln ^{\lambda_{2}-1}\left(V_{n}-\beta\right)}{\ln \left[\left(U_{m}-\alpha\right)\left(V_{n}-\beta\right)\right]}\right. \\
& \left.+\frac{1-\lambda_{2}}{\left(V_{n}-\beta\right)^{2-\lambda_{2}}}\right\} \frac{v_{n}}{\left(V_{n}-\beta\right) \ln ^{\lambda}\left[\left(U_{m}-\alpha\right)\left(V_{n}-\beta\right)\right]} .
\end{aligned}
$$

In the same way, for $x \in\left(n, n+\frac{1}{2}\right)$, we find

$$
\begin{aligned}
h^{\prime}(x)= & -\left\{\frac{\ln ^{\lambda_{2}-1}(V(x)-\beta)}{(V(x)-\beta)}+\frac{\lambda \ln ^{\lambda_{2}-1}(V(x)-\beta)}{\ln \left[\left(U_{m}-\alpha\right)(V(x)-\beta)\right]}\right. \\
& \left.+\frac{1-\lambda_{2}}{(V(x)-\beta)^{2-\lambda_{2}}}\right\} \frac{v_{n+1}}{(V(x)-\beta) \ln ^{\lambda}\left[\left(U_{m}-\alpha\right)(V(x)-\beta)\right]},
\end{aligned}
$$

$h^{\prime}(x)(<0)$ is strictly increasing in $\left(n, n+\frac{1}{2}\right)$ and

$$
\begin{aligned}
\lim _{x \rightarrow n+} h^{\prime}(x)= & h^{\prime}(n+0)=-\left\{\frac{\ln ^{\lambda_{2}-1}\left(V_{n}-\beta\right)}{\left(V_{n}-\beta\right)}+\frac{\lambda \ln ^{\lambda_{2}-1}\left(V_{n}-\beta\right)}{\ln \left[\left(U_{m}-\alpha\right)\left(V_{n}-\beta\right)\right]}\right. \\
& \left.+\frac{1-\lambda_{2}}{\left(V_{n}-\beta\right)^{2-\lambda_{2}}}\right\} \frac{v_{n+1}}{\left(V_{n}-\beta\right) \ln ^{\lambda}\left[\left(U_{m}-\alpha\right)\left(V_{n}-\beta\right)\right]} .
\end{aligned}
$$


Since $v_{n+1} \leq v_{n}$, we have $h^{\prime}(n-0) \leq h^{\prime}(n+0)$. Then by (9), for $m, n \in \mathbf{N} \backslash\{1\}$, it follows that

$$
h(n)<\int_{n-\frac{1}{2}}^{n+\frac{1}{2}} h(x) d x=\int_{n-\frac{1}{2}}^{n+\frac{1}{2}} \frac{\ln ^{\lambda_{2}-1}(V(x)-\beta)}{(V(x)-\beta)\left[\ln \left(U_{m}-\alpha\right)+\ln (V(x)-\beta)\right]^{\lambda}} d x .
$$

Lemma 2 For $m, n \in \mathbf{N} \backslash\{1\}$, we define the following weight coefficients:

$$
\begin{aligned}
& \omega\left(\lambda_{2}, m\right):=\sum_{n=2}^{\infty} \frac{1}{\ln ^{\lambda}\left[\left(U_{m}-\alpha\right)\left(V_{n}-\beta\right)\right]} \frac{v_{n+1} \ln ^{\lambda_{1}}\left(U_{m}-\alpha\right)}{\left(V_{n}-\beta\right) \ln ^{1-\lambda_{2}}\left(V_{n}-\beta\right)}, \\
& \varpi\left(\lambda_{1}, n\right):=\sum_{m=2}^{\infty} \frac{1}{\ln ^{\lambda^{\lambda}}\left[\left(U_{m}-\alpha\right)\left(V_{n}-\beta\right)\right]} \frac{\mu_{m+1} \ln ^{\lambda_{2}}\left(V_{n}-\beta\right)}{\left(U_{m}-\alpha\right) \ln ^{1-\lambda_{1}}\left(U_{m}-\alpha\right)} .
\end{aligned}
$$

If $\left\{\mu_{m}\right\}_{m=1}^{\infty}$ and $\left\{v_{n}\right\}_{n=1}^{\infty}$ are decreasing, and $U(\infty)=V(\infty)=\infty$, then

$$
\begin{array}{ll}
\omega\left(\lambda_{2}, m\right)<B\left(\lambda_{1}, \lambda_{2}\right) & (m \in \mathbf{N} \backslash\{1\}), \\
\varpi\left(\lambda_{1}, n\right)<B\left(\lambda_{1}, \lambda_{2}\right) & (n \in \mathbf{N} \backslash\{1\}) .
\end{array}
$$

Proof For $x \in\left(n-\frac{1}{2}, n+\frac{1}{2}\right) \backslash\{n\}, v_{n+1} \leq V^{\prime}(x)$, by (11), we obtain

$$
\begin{aligned}
\omega\left(\lambda_{2}, m\right) & <\sum_{n=2}^{\infty} v_{n+1} \int_{n-\frac{1}{2}}^{n+\frac{1}{2}} \frac{\ln ^{\lambda_{1}}\left(U_{m}-\alpha\right) \ln ^{\lambda_{2}-1}(V(x)-\beta) d x}{(V(x)-\beta)\left[\ln \left(U_{m}-\alpha\right)+\ln (V(x)-\beta)\right]^{\lambda}} \\
& \leq \sum_{n=2}^{\infty} \int_{n-\frac{1}{2}}^{n+\frac{1}{2}} \frac{\ln ^{\lambda_{1}}\left(U_{m}-\alpha\right) \ln ^{\lambda_{2}-1}(V(x)-\beta)}{\left[\ln \left(U_{m}-\alpha\right)+\ln (V(x)-\beta)\right]^{\lambda}} \frac{V^{\prime}(x)}{V(x)-\beta} d x \\
& =\int_{\frac{3}{2}}^{\infty} \frac{\ln ^{\lambda_{1}}\left(U_{m}-\alpha\right) \ln ^{\lambda_{2}-1}(V(x)-\beta)}{\left[\ln \left(U_{m}-\alpha\right)+\ln (V(x)-\beta)\right]^{\lambda}} \frac{V^{\prime}(x)}{V(x)-\beta} d x .
\end{aligned}
$$

Setting $t=\frac{\ln (V(x)-\beta)}{\ln \left(U_{m}-\alpha\right)}$, since $V\left(\frac{3}{2}\right)-\beta=1+\frac{v_{2}}{2}-\beta \geq 1$ and $\frac{V^{\prime}(x)}{V(x)-\beta} d x=\ln \left(U_{m}-\alpha\right) d t$, we find

$$
\omega\left(\lambda_{2}, m\right)<\int_{0}^{\infty} \frac{1}{(1+t)^{\lambda}} t^{\lambda_{2}-1} d t=B\left(\lambda_{1}, \lambda_{2}\right) .
$$

Hence, we obtain (14). In the same way, we obtain (15).

Lemma 3 Suppose that $\left\{\mu_{m}\right\}_{m=1}^{\infty}$ and $\left\{v_{n}\right\}_{n=1}^{\infty}$ are decreasing, and $U(\infty)=V(\infty)=\infty$. (i) For $m, n \in \mathbf{N} \backslash\{1\}$, we have

$$
\begin{aligned}
& B\left(\lambda_{1}, \lambda_{2}\right)\left(1-\theta\left(\lambda_{2}, m\right)\right)<\omega\left(\lambda_{2}, m\right), \\
& B\left(\lambda_{1}, \lambda_{2}\right)\left(1-\vartheta\left(\lambda_{1}, n\right)\right)<\varpi\left(\lambda_{1}, n\right),
\end{aligned}
$$

where

$$
\begin{aligned}
\theta\left(\lambda_{2}, m\right) & =\frac{1}{B\left(\lambda_{1}, \lambda_{2}\right)} \frac{\ln ^{\lambda_{2}-1}\left(1+v_{2}-\beta\right)}{\lambda_{2}\left[1+\frac{\ln \left(1+v_{2} \theta(m)-\beta\right)}{\ln \left(U_{m}-\alpha\right)}\right]^{\lambda}} \frac{1}{\ln ^{\lambda_{2}}\left(U_{m}-\alpha\right)} \\
& =O\left(\frac{1}{\ln ^{\lambda_{2}}\left(U_{m}-\alpha\right)}\right) \in(0,1) \quad\left(\theta(m) \in\left(\frac{\beta}{v_{2}}, 1\right)\right),
\end{aligned}
$$




$$
\begin{aligned}
\vartheta\left(\lambda_{1}, n\right) & =\frac{1}{B\left(\lambda_{1}, \lambda_{2}\right)} \frac{\ln ^{\lambda_{1}}\left(1+\mu_{2}-\alpha\right)}{\lambda_{1}\left[1+\frac{\ln \left(1+\mu_{2} \vartheta(n)-\alpha\right)}{\ln \left(V_{n}-\beta\right)}\right]^{\lambda}} \frac{1}{\ln ^{\lambda_{1}}\left(V_{n}-\beta\right)} \\
& =O\left(\frac{1}{\ln ^{\lambda_{1}}\left(V_{n}-\beta\right)}\right) \in(0,1) \quad\left(\vartheta(n) \in\left(\frac{\alpha}{\mu_{2}}, 1\right)\right) ;
\end{aligned}
$$

(ii) for any $c>0$, we have

$$
\begin{aligned}
& \sum_{m=2}^{\infty} \frac{\mu_{m+1}}{\left(U_{m}-\alpha\right) \ln ^{1+c}\left(U_{m}-\alpha\right)}=\frac{1}{c}\left(\frac{1}{\ln ^{c}\left(1+\mu_{2}-\alpha\right)}+c O(1)\right), \\
& \sum_{n=2}^{\infty} \frac{v_{n+1}}{\left(V_{n}-\beta\right) \ln ^{1+c}\left(V_{n}-\beta\right)}=\frac{1}{c}\left(\frac{1}{\ln ^{c}\left(1+v_{2}-\beta\right)}+c \widetilde{O}(1)\right) .
\end{aligned}
$$

Proof In view of $0 \leq \beta \leq \frac{v_{2}}{2}<v_{2}$, it follows that $\frac{\beta}{v_{2}}+1 \geq 1$ and $\frac{\beta}{v_{2}}+1<2$. By Example 1 , $h(x)$ is strictly decreasing in $[n, n+1]$, then for $m \in \mathbf{N} \backslash\{1\}$, we obtain

$$
\begin{aligned}
\omega\left(\lambda_{2}, m\right)> & \sum_{n=2}^{\infty} \int_{n}^{n+1} \frac{\ln ^{\lambda_{1}}\left(U_{m}-\alpha\right) \ln ^{\lambda_{2}-1}(V(x)-\beta) v_{n+1} d x}{(V(x)-\beta)\left[\ln \left(U_{m}-\alpha\right)+\ln (V(x)-\beta)\right]^{\lambda}} \\
= & \int_{2}^{\infty} \frac{\ln ^{\lambda_{1}}\left(U_{m}-\alpha\right) \ln ^{\lambda_{2}-1}(V(x)-\beta)}{\left[\ln \left(U_{m}-\alpha\right)+\ln (V(x)-\beta)\right]^{\lambda}} \frac{V^{\prime}(x)}{V(x)-\beta} d x \\
= & \int_{\frac{\beta}{v_{2}}+1}^{\infty} \frac{\ln ^{\lambda_{1}}\left(U_{m}-\alpha\right) \ln ^{\lambda_{2}-1}(V(x)-\beta)}{\left[\ln \left(U_{m}-\alpha\right)+\ln (V(x)-\beta)\right]^{\lambda}} \frac{V^{\prime}(x)}{V(x)-\beta} d x \\
& -\int_{\frac{\beta}{v_{2}}+1}^{2} \frac{\ln ^{\lambda_{1}}\left(U_{m}-\alpha\right) \ln \ln ^{\lambda_{2}-1}(V(x)-\beta)}{\left[\ln \left(U_{m}-\alpha\right)+\ln (V(x)-\beta)\right]^{\lambda}} \frac{V^{\prime}(x) d x}{V(x)-\beta} .
\end{aligned}
$$

Setting $t=\frac{\ln (V(x)-\beta)}{\ln \left(U_{m}-\alpha\right)}$, since

$$
\ln \left(V\left(\frac{\beta}{v_{2}}+1\right)-\beta\right)=\ln \left(1+v_{2} \frac{\beta}{v_{2}}-\beta\right)=0
$$

we find

$$
\begin{aligned}
\omega\left(\lambda_{2}, m\right) & >\int_{0}^{\infty} \frac{1}{(1+t)^{\lambda}} t^{\lambda_{2}-1} d t-\int_{\frac{\beta}{v_{2}}+1}^{2} \frac{\ln ^{\lambda_{1}}\left(U_{m}-\alpha\right) \ln ^{\lambda_{2}-1}(V(x)-\beta)}{\left[\ln \left(U_{m}-\alpha\right)+\ln (V(x)-\beta)\right]^{\lambda}} \frac{V^{\prime}(x)}{V(x)-\beta} d x \\
& =B\left(\lambda_{1}, \lambda_{2}\right)\left(1-\theta\left(\lambda_{2}, m\right)\right),
\end{aligned}
$$

where

$$
\theta\left(\lambda_{2}, m\right):=\frac{\ln ^{\lambda_{1}}\left(U_{m}-\alpha\right)}{B\left(\lambda_{1}, \lambda_{2}\right)} \int_{\frac{\beta}{v_{2}}+1}^{2} \frac{V^{\prime}(x) \ln ^{\lambda_{2}-1}(V(x)-\beta) d x}{(V(x)-\beta) \ln ^{\lambda}\left[\left(U_{m}-\alpha\right)(V(x)-\beta)\right]} \in(0,1) .
$$

In view of the integral mid value theorem, there exists a $\theta(m) \in\left(\frac{\beta}{v_{2}}, 1\right)$, satisfying

$$
\begin{aligned}
\theta\left(\lambda_{2}, m\right)= & \frac{1}{B\left(\lambda_{1}, \lambda_{2}\right)} \frac{\ln ^{\lambda_{1}}\left(U_{m}-\alpha\right) \ln ^{\lambda_{2}-1}(V(x)-\beta)}{\left[\ln \left(U_{m}-\alpha\right)+\ln (V(1+\theta(m))-\beta)\right]^{\lambda}} \\
& \times \int_{\frac{\beta}{v_{2}}+1}^{2} \ln ^{\lambda_{2}-1}(V(x)-\beta) \frac{V^{\prime}(x)}{V(x)-\beta} d x
\end{aligned}
$$




$$
\begin{aligned}
& =\frac{1}{B\left(\lambda_{1}, \lambda_{2}\right)} \frac{\ln ^{\lambda_{1}}\left(U_{m}-\alpha\right) \ln ^{\lambda_{2}-1}\left(1+v_{2}-\beta\right)}{\left[\ln \left(U_{m}-\alpha\right)+\ln \left(1+v_{2} \theta(m)-\beta\right)\right]^{\lambda}} \\
& =\frac{1}{B\left(\lambda_{1}, \lambda_{2}\right)} \frac{\ln ^{\lambda_{2}-1}\left(1+v_{2}-\beta\right)}{\lambda_{2}\left[1+\frac{\ln \left(1+v_{2} \theta(m)-\beta\right)}{\ln \left(U_{m}-\alpha\right)}\right]^{\lambda}} \frac{1}{\ln ^{\lambda_{2}}\left(U_{m}-\alpha\right)} .
\end{aligned}
$$

Since we find

$$
0<\theta\left(\lambda_{2}, m\right) \leq \frac{\ln ^{\lambda_{2}-1}\left(1+v_{2}-\beta\right)}{\lambda_{2}} \frac{1}{\ln ^{\lambda_{2}}\left(U_{m}-\alpha\right)},
$$

namely, $\theta\left(\lambda_{2}, m\right)=O\left(\frac{1}{\ln ^{\lambda} 2\left(U_{m}-\alpha\right)}\right)$, we have (16) and (18). In the same way, we obtain (17) and (19).

For any $c>0$, it follows that

$$
\begin{aligned}
& \sum_{m=2}^{\infty} \frac{\mu_{m+1}}{\left(U_{m}-\alpha\right) \ln ^{1+c}\left(U_{m}-\alpha\right)} \\
& \leq \sum_{m=2}^{\infty} \frac{\mu_{m}}{\left(U_{m}-\alpha\right) \ln ^{1+c}\left(U_{m}-\alpha\right)} \\
& =\frac{\mu_{2}}{\left(U_{2}-\alpha\right) \ln ^{1+c}\left(U_{2}-\alpha\right)}+\sum_{m=3}^{\infty} \frac{\mu_{m}}{\left(U_{m}-\alpha\right) \ln ^{1+c}\left(U_{m}-\alpha\right)} \\
& =\frac{\mu_{2}}{\left(U_{2}-\alpha\right) \ln ^{1+c}\left(U_{2}-\alpha\right)}+\sum_{m=3}^{\infty} \int_{m-1}^{m} \frac{U^{\prime}(x) d x}{\left(U_{m}-\alpha\right) \ln ^{1+c}\left(U_{m}-\alpha\right)} \\
& <\frac{\mu_{2}}{\left(U_{2}-\alpha\right) \ln ^{1+c}\left(U_{2}-\alpha\right)}+\sum_{m=3}^{\infty} \int_{m-1}^{m} \frac{U^{\prime}(x) d x}{(U(x)-\alpha) \ln ^{1+c}(U(x)-\alpha)} \\
& =\frac{\mu_{2}}{\left(U_{2}-\alpha\right) \ln ^{1+c}\left(U_{2}-\alpha\right)}+\int_{2}^{\infty} \frac{U^{\prime}(x) d x}{(U(x)-\alpha) \ln ^{1+c}(U(x)-\alpha)} \\
& =\frac{\mu_{2}}{\left(U_{2}-\alpha\right) \ln ^{1+c}\left(U_{2}-\alpha\right)}+\frac{1}{c \ln ^{c}\left(1+\mu_{2}-\alpha\right)} \\
& =\frac{1}{c}\left[\frac{1}{\ln ^{c}\left(1+\mu_{2}-\alpha\right)}+\frac{c \mu_{2}}{\left(U_{2}-\alpha\right) \ln ^{1+c}\left(U_{2}-\alpha\right)}\right] \text {, } \\
& \sum_{m=2}^{\infty} \frac{\mu_{m+1}}{\left(U_{m}-\alpha\right) \ln ^{1+c}\left(U_{m}-\alpha\right)} \\
& =\sum_{m=2}^{\infty} \int_{m}^{m+1} \frac{U^{\prime}(x) d x}{\left(U_{m}-\alpha\right) \ln ^{1+c}\left(U_{m}-\alpha\right)} \\
& >\sum_{m=2}^{\infty} \int_{m}^{m+1} \frac{U^{\prime}(x)}{(U(x)-\alpha) \ln ^{1+c}(U(x)-\alpha)} d x \\
& =\int_{2}^{\infty} \frac{U^{\prime}(x) d x}{(U(x)-\alpha) \ln ^{1+c}(U(x)-\alpha)}=\frac{1}{c \ln ^{c}\left(1+\mu_{2}-\alpha\right)} .
\end{aligned}
$$

Hence we obtain (20). In the same way, we obtain (21). 


\section{Main results}

We define the following functions:

$$
\begin{aligned}
& \widetilde{\Phi}_{\lambda}(m):=\omega\left(\lambda_{2}, m\right) \frac{\left[\ln \left(U_{m}-\alpha\right)\right]^{p\left(1-\lambda_{1}\right)-1}}{\left(U_{m}-\alpha\right)^{1-p} \mu_{m+1}^{p-1}} \\
& \widetilde{\Psi}_{\lambda}(n):=\varpi\left(\lambda_{1}, n\right) \frac{\left[\ln \left(V_{n}-\beta\right)\right]^{q\left(1-\lambda_{2}\right)-1}}{\left(V_{n}-\beta\right)^{1-q} v_{n+1}^{q-1}} \quad(m, n \in \mathbf{N} \backslash\{1\}) .
\end{aligned}
$$

Theorem 1 We have the following equivalent inequalities:

$$
\begin{aligned}
& I:=\sum_{n=2}^{\infty} \sum_{m=2}^{\infty} \frac{a_{m} b_{n}}{\ln ^{\lambda}\left[\left(U_{m}-\alpha\right)\left(V_{n}-\beta\right)\right]} \leq\|a\|_{p, \widetilde{\Phi}_{\lambda}}\|b\|_{q, \widetilde{\Psi}_{\lambda},} \\
& J:=\left\{\sum_{n=2}^{\infty} \frac{v_{n+1} \ln ^{p \lambda_{2}-1}\left(V_{n}-\beta\right)}{\left(\varpi\left(\lambda_{1}, n\right)\right)^{p-1}\left(V_{n}-\beta\right)}\left[\sum_{m=2}^{\infty} \frac{a_{m}}{\ln ^{\lambda}\left[\left(U_{m}-\alpha\right)\left(V_{n}-\beta\right)\right]}\right]^{p}\right\}^{\frac{1}{p}} \leq\|a\|_{p, \widetilde{\Phi}_{\lambda}} .
\end{aligned}
$$

Proof By Hölder's inequality (cf. [15]) and (13), we find

$$
\begin{aligned}
{\left[\sum_{m=2}^{\infty}\right.} & \left.\frac{a_{m}}{\ln ^{\lambda}\left[\left(U_{m}-\alpha\right)\left(V_{n}-\beta\right)\right]}\right]^{p} \\
= & {\left[\sum_{m=2}^{\infty} \frac{1}{\ln ^{\lambda}\left[\left(U_{m}-\alpha\right)\left(V_{n}-\beta\right)\right]}\right.} \\
& \left.\times\left(\frac{\left(U_{m}-\alpha\right)^{1 / q} \ln ^{\left(1-\lambda_{1}\right) / q}\left(U_{m}-\alpha\right)}{\mu_{m+1}^{1 / q} \ln ^{\left(1-\lambda_{2}\right) / p}\left(V_{n}-\beta\right)} a_{m}\right)\left(\frac{\mu_{m+1}^{1 / q} \ln ^{\left(1-\lambda_{2}\right) / p}\left(V_{n}-\beta\right)}{\left(U_{m}-\alpha\right)^{1 / q} \ln ^{\left(1-\lambda_{1}\right) / q}\left(U_{m}-\alpha\right)}\right)\right]^{p} \\
\leq & \sum_{m=2}^{\infty} \frac{1}{\ln ^{\lambda}\left[\left(U_{m}-\alpha\right)\left(V_{n}-\beta\right)\right]} \frac{\left(U_{m}-\alpha\right)^{p-1} \ln ^{\left(1-\lambda_{1}\right) p / q}\left(U_{m}-\alpha\right)}{\mu_{m+1}^{p / q} \ln ^{1-\lambda_{2}}\left(V_{n}-\beta\right)} a_{m}^{p} \\
& \times\left[\sum_{m=2}^{\infty} \frac{\mu_{m+1}}{\ln ^{\lambda}\left[\left(U_{m}-\alpha\right)\left(V_{n}-\beta\right)\right]} \frac{\ln ^{\left(1-\lambda_{2}\right)(q-1)}\left(V_{n}-\beta\right)}{\left(U_{m}-\alpha\right) \ln ^{1-\lambda_{1}}\left(U_{m}-\alpha\right)}\right]^{p-1} \\
= & \frac{\left.\left(\varpi \lambda_{1}, n\right)\right)^{p-1}\left(V_{n}-\beta\right)}{v_{n+1} \ln ^{p \lambda_{2}-1}\left(V_{n}-\beta\right)} \\
& \times \sum_{m=2}^{\infty} \frac{v_{n+1}\left(U_{m}-\alpha\right)^{p-1} \ln ^{\left(1-\lambda_{1}\right)(p-1)}\left(U_{m}-\alpha\right)}{\mu_{m+1}^{p-1}\left(V_{n}-\beta\right) \ln ^{\lambda}\left[\left(U_{m}-\alpha\right)\left(V_{n}-\beta\right)\right] \ln ^{1-\lambda_{2}}\left(V_{n}-\beta\right)} a_{m}^{p} .
\end{aligned}
$$

Then by (12) we obtain

$$
\begin{aligned}
J & \leq\left[\sum_{n=2}^{\infty} \sum_{m=2}^{\infty} \frac{v_{n+1}\left(U_{m}-\alpha\right)^{p-1} a_{m}^{p}}{\ln ^{\lambda}\left[\left(U_{m}-\alpha\right)\left(V_{n}-\beta\right)\right]} \frac{\ln ^{\left(1-\lambda_{1}\right)(p-1)}\left(U_{m}-\alpha\right)}{\mu_{m+1}^{p-1}\left(V_{n}-\beta\right) \ln ^{1-\lambda_{2}}\left(V_{n}-\beta\right)}\right]^{\frac{1}{p}} \\
& =\left[\sum_{m=2}^{\infty} \sum_{n=2}^{\infty} \frac{v_{n+1}\left(U_{m}-\alpha\right)^{p-1} a_{m}^{p}}{\ln ^{\lambda}\left[\left(U_{m}-\alpha\right)\left(V_{n}-\beta\right)\right]} \frac{\ln ^{\left(1-\lambda_{1}\right)(p-1)}\left(U_{m}-\alpha\right)}{\mu_{m+1}^{p-1}\left(V_{n}-\beta\right) \ln ^{1-\lambda_{2}}\left(V_{n}-\beta\right)}\right]^{\frac{1}{p}} \\
& =\left[\sum_{m=2}^{\infty} \omega\left(\lambda_{2}, m\right) \frac{\ln ^{p\left(1-\lambda_{1}\right)-1}\left(U_{m}-\alpha\right)}{\left(U_{m}-\alpha\right)^{1-p} \mu_{m+1}^{p-1}} a_{m}^{p}\right]^{\frac{1}{p}}
\end{aligned}
$$


namely, (24) follows. By Hölder's inequality (cf. [15]), we find

$$
\begin{aligned}
I= & \sum_{n=2}^{\infty}\left[\frac{v_{n+1}^{1 / p} \ln ^{\lambda_{2}-\frac{1}{p}}\left(V_{n}-\beta\right)}{\left(\varpi\left(\lambda_{1}, n\right)\right)^{\frac{1}{q}}\left(V_{n}-\beta\right)^{1 / p}} \sum_{m=1}^{\infty} \frac{a_{m}}{\ln ^{\lambda}\left[\left(U_{m}-\alpha\right)\left(V_{n}-\beta\right)\right]}\right] \\
& \times\left[\left(\varpi\left(\lambda_{1}, n\right)\right)^{\frac{1}{q}} \frac{\ln ^{\frac{1}{p}-\lambda_{2}}\left(V_{n}-\beta\right)}{\left(V_{n}-\beta\right)^{-1 / p} v_{n+1}^{1 / p}} b_{n}\right] \leq J\|b\|_{q, \widetilde{\Psi}_{\lambda}} .
\end{aligned}
$$

Then by (24), (23) follows.

On the other hand, suppose that (23) is valid. We set

$$
b_{n}:=\frac{v_{n+1} \ln ^{p \lambda_{2}-1}\left(V_{n}-\beta\right)}{\left(\varpi\left(\lambda_{1}, n\right)\right)^{p-1}\left(V_{n}-\beta\right)}\left[\sum_{m=2}^{\infty} \frac{a_{m}}{\ln ^{\lambda}\left[\left(U_{m}-\alpha\right)\left(V_{n}-\beta\right)\right]}\right]^{p-1}, \quad n \in \mathbf{N} \backslash\{1\} .
$$

Then we have $J^{p}=\|b\|_{q, \widetilde{\Psi}_{\lambda}}^{q}$. If $J=0$, then (24) is trivially valid; if $J=\infty$, then in view of (26), (24) takes the form of an equality. Suppose that $0<J<\infty$. By (23), we obtain

$$
\begin{aligned}
& \|b\|_{q, \widetilde{\Psi}_{\lambda}}^{q}=J^{p}=I \leq\|a\|_{p, \widetilde{\Phi}_{\lambda}}\|b\|_{q, \widetilde{\Psi}_{\lambda}}, \\
& \|b\|_{q, \widetilde{\Psi}_{\lambda}}^{q-1}=J \leq\|a\|_{p, \widetilde{\Phi}_{\lambda}},
\end{aligned}
$$

namely, (24) follows, which is equivalent to (23).

Theorem 2 Assuming that $\left\{\mu_{m}\right\}_{m=1}^{\infty}$ and $\left\{v_{n}\right\}_{n=1}^{\infty}$ are decreasing, $U(\infty)=V(\infty)=\infty, 0<$ $\|a\|_{p, \Phi_{\lambda}},\|b\|_{q, \Psi_{\lambda}}<\infty$, we have the following equivalent inequalities:

$$
\begin{aligned}
\sum_{n=2}^{\infty} \sum_{m=2}^{\infty} \frac{a_{m} b_{n}}{\ln ^{\lambda}\left[\left(U_{m}-\alpha\right)\left(V_{n}-\beta\right)\right]}<B\left(\lambda_{1}, \lambda_{2}\right)\|a\|_{p, \Phi_{\lambda}}\|b\|_{q, \Psi_{\lambda},}, \\
J_{1}:=\left\{\sum_{n=2}^{\infty} \frac{v_{n+1} \ln ^{p \lambda_{2}-1}\left(V_{n}-\beta\right)}{V_{n}-\beta}\right. \\
\times\left[\sum_{m=2}^{\infty} \frac{a_{m}}{\ln ^{\lambda}\left[\left(U_{m}-\alpha\right)\left(V_{n}-\beta\right)\right]}\right]^{p}<B\left(\lambda_{1}, \lambda_{2}\right)\|a\|_{p, \Phi_{\lambda}},
\end{aligned}
$$

where the constant factor $B\left(\lambda_{1}, \lambda_{2}\right)$ is the best possible.

Proof Applying (14) and (15) in (23) and (24), we have the equivalent inequalities (31) and (32).

$$
\begin{aligned}
& \text { For } \varepsilon \in\left(0, p \lambda_{1}\right) \text {, we set } \tilde{\lambda}_{1}=\lambda_{1}-\frac{\varepsilon}{p}(\in(0,1)), \tilde{\lambda}_{2}=\lambda_{2}+\frac{\varepsilon}{p}(>0) \text {, and } \\
& \tilde{a}_{m}:=\frac{\mu_{m+1}}{U_{m}-\alpha} \ln ^{\tilde{\lambda}_{1}-1}\left(U_{m}-\alpha\right), \quad \tilde{b}_{n}:=\frac{v_{n+1}}{V_{n}-\beta} \ln ^{\tilde{\lambda}_{2}-\varepsilon-1}\left(V_{n}-\beta\right) .
\end{aligned}
$$


Then by (20), (21), and (17), we obtain

$$
\begin{aligned}
& \|\widetilde{a}\|_{p, \Phi_{\lambda}}\|\widetilde{b}\|_{q, \Psi_{\lambda}} \\
& =\left[\sum_{m=2}^{\infty} \frac{\mu_{m+1}}{\left(U_{m}-\alpha\right) \ln ^{1+\varepsilon}\left(U_{m}-\alpha\right)}\right]^{\frac{1}{p}}\left[\sum_{n=2}^{\infty} \frac{v_{n+1}}{\left(V_{n}-\beta\right) \ln ^{1+\varepsilon}\left(V_{n}-\beta\right)}\right]^{\frac{1}{q}} \\
& =\frac{1}{\varepsilon}\left[\frac{1}{\ln ^{\varepsilon}\left(1+\mu_{2}-\alpha\right)}+\varepsilon O(1)\right]^{\frac{1}{p}}\left[\frac{1}{\ln ^{\varepsilon}\left(1+v_{2}-\beta\right)}+\varepsilon \widetilde{O}(1)\right]^{\frac{1}{q}}, \\
& \widetilde{I}:=\sum_{n=2}^{\infty} \sum_{m=2}^{\infty} \frac{\widetilde{a}_{m} \widetilde{b}_{n}}{\ln ^{\lambda}\left[\left(U_{m}-\alpha\right)\left(V_{n}-\beta\right)\right]} \\
& =\sum_{n=2}^{\infty}\left[\sum_{m=2}^{\infty} \frac{1}{\ln ^{\lambda}\left[\left(U_{m}-\alpha\right)\left(V_{n}-\beta\right)\right]} \frac{\mu_{m+1} \ln ^{\tilde{\lambda}_{2}}\left(V_{n}-\beta\right)}{\left(U_{m}-\alpha\right) \ln ^{1-\tilde{\lambda}_{1}}\left(U_{m}-\alpha\right)}\right] \\
& \times \frac{v_{n+1}}{\left(V_{n}-\beta\right) \ln ^{\varepsilon+1}\left(V_{n}-\beta\right)}=\sum_{n=2}^{\infty} \varpi\left(\tilde{\lambda}_{1}, n\right) \frac{v_{n+1}}{\left(V_{n}-\beta\right) \ln ^{\varepsilon+1}\left(V_{n}-\beta\right)} \\
& \geq B\left(\tilde{\lambda}_{1}, \tilde{\lambda}_{2}\right)\left[\sum_{n=2}^{\infty} \frac{v_{n+1}}{\left(V_{n}-\beta\right) \ln ^{\varepsilon+1}\left(V_{n}-\beta\right)}\right. \\
& \left.-\sum_{n=2}^{\infty} O\left(\frac{v_{n+1}}{\left(V_{n}-\beta\right) \ln ^{\lambda_{1}+\frac{\varepsilon}{q}+1}\left(V_{n}-\beta\right)}\right)\right] \\
& =\frac{1}{\varepsilon} B\left(\tilde{\lambda}_{1}, \tilde{\lambda}_{2}\right)\left[\frac{1}{\ln ^{\varepsilon}\left(1+v_{2}-\beta\right)}+\varepsilon(\widetilde{O}(1)-O(1))\right] \text {. }
\end{aligned}
$$

If there exists a positive constant $K \leq B\left(\lambda_{1}, \lambda_{2}\right)$, such that (31) is valid when replacing $B\left(\lambda_{1}, \lambda_{2}\right)$ by $K$, then in particular, we have $\varepsilon \widetilde{I}<\varepsilon K\|\widetilde{a}\|_{p, \Phi_{\lambda}}\|\widetilde{b}\|_{q, \Psi_{\lambda}}$, namely

$$
\begin{aligned}
& B\left(\lambda_{1}-\frac{\varepsilon}{p}, \lambda_{2}+\frac{\varepsilon}{p}\right)\left[\frac{1}{\ln ^{\varepsilon}\left(1+v_{2}-\beta\right)}+\varepsilon(\widetilde{O}(1)-O(1))\right] \\
& \quad<K\left[\frac{1}{\ln ^{\varepsilon}\left(1+\mu_{2}-\alpha\right)}+\varepsilon O(1)\right]^{\frac{1}{p}}\left[\frac{1}{\ln ^{\varepsilon}\left(1+v_{2}-\beta\right)}+\varepsilon \widetilde{O}(1)\right]^{\frac{1}{q}} .
\end{aligned}
$$

It follows that $B\left(\lambda_{1}, \lambda_{2}\right) \leq K\left(\varepsilon \rightarrow 0^{+}\right)$. Hence, $K=B\left(\lambda_{1}, \lambda_{2}\right)$ is the best possible constant factor of (31).

Similarly, we can obtain

$$
I \leq J_{1}\|b\|_{q, \Psi_{\lambda}}
$$

Hence, we can prove that the constant factor $B\left(\lambda_{1}, \lambda_{2}\right)$ in (32) is the best possible. Otherwise, we would reach a contradiction by (34) that the constant factor in (31) is not the best possible.

We find $\Psi_{\lambda}^{1-p}(n)=\frac{v_{n+1}}{V_{n}-\beta} \ln ^{p \lambda_{2}-1}\left(V_{n}-\beta\right)$, and we define the following weighted normed spaces:

$$
l_{p, \Phi_{\lambda}}:=\left\{a=\left\{a_{m}\right\}_{m=2}^{\infty} ;\|a\|_{p, \Phi_{\lambda}}<\infty\right\}
$$




$$
\begin{aligned}
& l_{q, \Psi_{\lambda}}:=\left\{b=\left\{b_{n}\right\}_{n=2}^{\infty} ;\|b\|_{q, \Psi_{\lambda}}<\infty\right\}, \\
& l_{p, \Psi_{\lambda}^{1-p}}:=\left\{c=\left\{c_{n}\right\}_{n=2}^{\infty} ;\|c\|_{p, \Psi_{\lambda}^{1-p}}<\infty\right\} .
\end{aligned}
$$

Assuming that $a=\left\{a_{m}\right\}_{m=2}^{\infty} \in l_{p, \Phi_{\lambda}}$, setting

$$
c=\left\{c_{n}\right\}_{n=2}^{\infty}, \quad c_{n}:=\sum_{m=2}^{\infty} \frac{a_{m}}{\ln ^{\lambda}\left[\left(U_{m}-\alpha\right)\left(V_{n}-\beta\right)\right]}, \quad n \in \mathbf{N} \backslash\{1\},
$$

we can rewrite (32) as follows:

$$
\|c\|_{p, \Psi_{\lambda}^{1-p}}<B\left(\lambda_{1}, \lambda_{2}\right)\|a\|_{p, \Phi_{\lambda}}<\infty
$$

namely, $c \in l_{p, \Psi_{\lambda}^{1-p}}$.

Definition 1 Define a Hardy-Mulholland-type operator $T: l_{p, \Phi_{\lambda}} \rightarrow l_{p, \Psi_{\lambda}^{1-p}}$ as follows: For any $a=\left\{a_{m}\right\}_{m=2}^{\infty} \in l_{p, \Phi_{\lambda}}$, there exists a unique representation $T a=c \in l_{p, \Psi_{\lambda}^{1-p}}$. We set the formal inner product of $T a$ and $b=\left\{b_{n}\right\}_{n=2}^{\infty} \in l_{q, \Psi_{\lambda}}$ as follows:

$$
(T a, b):=\sum_{n=2}^{\infty}\left[\sum_{m=2}^{\infty} \frac{a_{m}}{\ln ^{\lambda}\left[\left(U_{m}-\alpha\right)\left(V_{n}-\beta\right)\right]}\right] b_{n} .
$$

Then we can rewrite (31) and (32) as follows:

$$
\begin{aligned}
& (T a, b)<B\left(\lambda_{1}, \lambda_{2}\right)\|a\|_{p, \Phi_{\lambda}}\|b\|_{q, \Psi_{\lambda}}, \\
& \|T a\|_{p, \Psi_{\lambda}^{1-p}}<B\left(\lambda_{1}, \lambda_{2}\right)\|a\|_{p, \Phi_{\lambda}} .
\end{aligned}
$$

We set the norm of operator $T$ as follows:

$$
\|T\|:=\sup _{a(\neq \theta) \in l_{p, \Phi_{\lambda}}} \frac{\|T a\|_{p, \Psi_{\lambda}^{1-p}}}{\|a\|_{p, \Phi_{\lambda}}} .
$$

By (37), we find $\|T\| \leq B\left(\lambda_{1}, \lambda_{2}\right)$. Since the constant factor in (37) is the best possible, it follows that $\|T\|=B\left(\lambda_{1}, \lambda_{2}\right)$.

Remark 1 (i) For $\alpha=\beta=0$ in (31) and (32), setting

$$
\varphi_{\lambda}(m):=\frac{\left(\ln U_{m}\right)^{p\left(1-\lambda_{1}\right)-1}}{U_{m}^{1-p} \mu_{m+1}^{p-1}}, \quad \psi_{\lambda}(n):=\frac{\left(\ln V_{n}\right)^{q\left(1-\lambda_{2}\right)-1}}{V_{n}^{1-q} v_{n+1}^{q-1}} \quad(m, n \in \mathbf{N} \backslash\{1\}),
$$

we have the following equivalent Hardy-Mulholland-type inequalities:

$$
\begin{aligned}
& \sum_{n=2}^{\infty} \sum_{m=2}^{\infty} \frac{a_{m} b_{n}}{\ln ^{\lambda}\left(U_{m} V_{n}\right)}<B\left(\lambda_{1}, \lambda_{2}\right)\|a\|_{p, \varphi_{\lambda}}\|b\|_{q, \psi_{\lambda}}, \\
& \left\{\sum_{n=2}^{\infty} \frac{v_{n+1}}{V_{n}} \ln ^{p \lambda_{2}-1} V_{n}\left[\sum_{m=2}^{\infty} \frac{a_{m}}{\ln ^{\lambda}\left(U_{m} V_{n}\right)}\right]^{p}\right\}^{\frac{1}{p}}<B\left(\lambda_{1}, \lambda_{2}\right)\|a\|_{p, \varphi_{\lambda}} .
\end{aligned}
$$


For $\lambda=1, \lambda_{1}=\frac{1}{q}, \lambda_{2}=\frac{1}{p}$ in (38) and (39), we have the following equivalent inequality:

$$
\begin{aligned}
& \sum_{n=2}^{\infty} \sum_{m=2}^{\infty} \frac{a_{m} b_{n}}{\ln \left(U_{m} V_{n}\right)}<\frac{\pi}{\sin \left(\frac{\pi}{p}\right)}\left[\sum_{m=2}^{\infty}\left(\frac{U_{m}}{\mu_{m+1}}\right)^{p-1} a_{m}^{p}\right]^{\frac{1}{p}}\left[\sum_{n=2}^{\infty}\left(\frac{V_{n}}{v_{n+1}}\right)^{q-1} b_{n}^{q}\right]^{\frac{1}{q}}, \\
& \left\{\sum_{n=2}^{\infty} \frac{v_{n+1}}{V_{n}}\left[\sum_{m=2}^{\infty} \frac{a_{m}}{\ln \left(U_{m} V_{n}\right)}\right]^{p}\right\}^{\frac{1}{p}}<\frac{\pi}{\sin \left(\frac{\pi}{p}\right)}\left[\sum_{m=2}^{\infty}\left(\frac{U_{m}}{\mu_{m+1}}\right)^{p-1} a_{m}^{p}\right]^{\frac{1}{p}} .
\end{aligned}
$$

Hence, (38) is an extension of (40), and (31) is a more accurate inequality of (38) (for $0<\alpha \leq \frac{\mu_{2}}{2}, 0<\beta \leq \frac{v_{2}}{2}$ ).

(ii) For $\mu_{i}=v_{j}=1(i, j \in \mathbf{N}), \lambda=1, \lambda_{1}=\frac{1}{q}, \lambda_{2}=\frac{1}{p}$ in (31), we reduce our case to the following inequality: For $\alpha, \beta \leq \frac{1}{2}$,

$$
\begin{aligned}
& \sum_{m=2}^{\infty} \sum_{n=2}^{\infty} \frac{a_{m} b_{n}}{\ln [(m-\alpha)(n-\beta)]} \\
& \quad<\frac{\pi}{\sin (\pi / p)}\left[\sum_{m=2}^{\infty} \frac{a_{m}^{p}}{(m-\alpha)^{1-p}}\right]^{\frac{1}{p}}\left[\sum_{n=2}^{\infty} \frac{b_{n}^{q}}{(n-\beta)^{1-q}}\right]^{\frac{1}{q}},
\end{aligned}
$$

Hence, (42) is a more accurate inequality of (3) (for $0<\alpha, \beta \leq \frac{1}{2}$ ).

\section{Competing interests}

The authors declare that they have no competing interests.

\section{Authors' contributions}

BY carried out the mathematical studies, participated in the sequence alignment and drafted the manuscript. AL and LH participated in the design of the study and performed the numerical analysis. All authors read and approved the final manuscript.

\section{Author details}

${ }^{1}$ College of Mathematics and Statistics, Jishou University, Jishou, Hunan 416000, P.R. China. ${ }^{2}$ Department of Mathematics, Guangdong University of Education, Guangzhou, Guangdong 51003, P.R. China.

\section{Acknowledgements}

This work is supported by Hunan Province Natural Science Foundation (No. 2015JJ4041), and the National Natural Science Foundation of China (No. 61370186). Thanks for their help.

Received: 7 December 2015 Accepted: 8 February 2016 Published online: 19 February 2016

\section{References}

1. Hardy, GH, Littlewood, JE, Pólya, G: Inequalities. Cambridge University Press, Cambridge (1934)

2. Yang, BC: Discrete Hilbert-Type Inequalities. Bentham Science Publishers, Sharjah (2011)

3. Mulholland, HP: Some theorems on Dirichlet series with positive coefficients and related integrals. Proc. Lond. Math. Soc. 29(2), 281-292 (1929)

4. Mitrinović, DS, Pečarić, JE, Fink, AM: Inequalities Involving Functions and Their Integrals and Derivatives. Kluwer Academic, Boston (1991)

5. Yang, BC: Hilbert-Type Integral Inequalities. Bentham Science Publishers, Sharjah (2009)

6. Yang, BC: On Hilbert's integral inequality. J. Math. Anal. Appl. 220, 778-785 (1998)

7. Yang, BC: An extension of Mulholland's inequality. Jordan J. Math. Stat. 3(3), 151-157 (2010)

8. Chen, Q, Yang, BC: A survey on the study of Hilbert-type inequalities. J. Inequal. Appl. 2015, 302 (2015)

9. Yang, BC: The Norm of Operator and Hilbert-Type Inequalities. Science Press, Beijing (2009) (China)

10. Yang, BC: An extension of a Hardy-Hilbert-type inequality. J. Guangdong Univ. Educ. 35(3), 1-7 (2015)

11. Wang, DX, Guo, DR: Introduction to Spectral Functions. Science Press, Beijing (1979) (China)

12. Huang, QL, Yang, BC: A more accurate Hardy-Hilbert-type inequality. J. Guangdong Univ. Educ. 35(5), 27-35 (2015)

13. Yang, BC, Chen, Q: On a Hardy-Hilbert-type inequality with parameters. J. Inequal. Appl. 2015, 339 (2015)

14. Wang, AZ, Huang, QL, Yang, BC: A strengthened Mulholland-type inequality with parameters. J. Inequal. Appl. 2015 $329(2015)$

15. Kuang, JC: Applied Inequalities. Shangdong Science Technic Press, Jinan (2004) 